\title{
Growth conditions influence on thermally stimulated luminescence of sapphire single crystals
}

\author{
I.V. Blonskyy ${ }^{1}$, A.Yu. Vakhnin ${ }^{1}$, A.Ya. Danko ${ }^{2}$, A.K. Kadashchuk ${ }^{1}$, V.N. Kadan ${ }^{1}$, \\ N.S. Sidelnikova ${ }^{2}$, V.M. Puzikov², Yu.A. Skryshevskiii ${ }^{1}$ \\ ${ }^{1}$ Institute of Physics, NAS of Ukraine, 46 prospect Nauky, 03028 Kyiv, Ukraine \\ Phone: 265 3138; e-mail: blon@iop.kiev.ua \\ ${ }^{2}$ Institute of single crystals, NAS of Ukraine, 60 prospect Lenina, 61001 Kharkov, Ukraine \\ Phone:(0572) 30 7485; e-mail:root@isc.kharkov.com
}

\begin{abstract}
The results of investigation of thermally stimulated and photoluminescence as well as absorption spectra of a-sapphire grown by a technique of horizontally directed crystallization in a protective gas medium of varying composition and pressure are presented. This technique has an advantage of minimization of $\mathrm{W}$ and Mo erosion, thus providing a considerably reduced prime cost. At the same time, there are some problems connected with emergence of considerable number of anion vacancies, whose in-axis concentration gradient is directed oppositely to that of dopants (mainly Ti). The correlations are found between the conditions of $\alpha$-sapphire growing and the data of absorption and luminescent spectroscopy. An interaction between the anion vacancies and Ti dopants, which induces a transition from electric-charge state $\mathrm{Ti}^{4+}$ to $\mathrm{Ti}^{3+}$, is discussed.
\end{abstract}

Keywords: sapphire, thermally stimulated luminescence, titanium dopant, anion vacancy, absorption, photoluminescence.

Paper received 23.07.02; accepted for publication 17.12.02.

\section{Introduction}

Last time the single crystalline sapphire $\left(\alpha-\mathrm{Al}_{2} \mathrm{O}_{3}\right)$ finds new fields of application. We will mention only those ones related to electronics. First of all sapphire is the basic substrate material for electronic devices, particularly light emitting diodes (LEDs). Today's priority is development of white LEDs, which in the outlook have to replace the less energy-effective incandescent lamps [1]. As a result of several design programs, the various working models of such devices have been elaborated and tested, and at the same time the influence of impurity and defect states in sapphire substrate on the spectrum and chromatic coordinate of the resulting radiation was revealed [2]. Attempts are going on to make active laser media on F-centers, which are genetically related with anion vacancies in sapphire [3]. The authors of [4] proposed to use the sapphire with F-centers in a new generation of dosimetric devices. The works are going to improve the beam strength of sapphire single crystal elements for high-power UV optics [5]. All the above examples show that the impurity/defect subsystem in the single crystalline sapphire plays a significant role in $\mathrm{Al}_{2} \mathrm{O}_{3}$ based electronic devices and its properties depend in its turn on the growth technology.
The Institute of single crystals of NASU for several years develops the basics of a "cheap" growth technology allowing to obtain the single crystalline sapphire of commercial quality in protective gaseous atmosphere $[6,8]$. One of the advantages of this technology is the minimal erosion of tungsten and molybdenum during the growth process. It is one of the reasons of its low prime cost. However the variation of growth conditions must be accompanied by the change in single crystalline impurity/defect subsystem. The thermally stimulated luminescence (TSL) is a well-known effective method for study of defect states of various nature. Taking into account the above considerations the aim of the present study is a search for correlations between the technological conditions of sapphire crystal growth and its TSL signal as well as analysis of the origin of TSL peaks. The sapphire samples grown both in gaseous ambient of various compositions ( $\mathrm{Ar}+\mathrm{CO}, \mathrm{CO}, \mathrm{CO}+\mathrm{O}_{2}, \mathrm{Ar}+$ air) under different pressure $\left(10^{-1} \div 100\right.$ torr $)$ and by common vacuum technology $\left(10^{-5}\right.$ torr $)$ have been studied.

\section{Experimental technique}

The sapphire single crystals were grown in a $10^{-4}$ torr vacuum or protective gas media of different composition 


\section{I.V. Blonskyy et al.: Growth conditions influence on thermally ...}

and pressure with using the graphitized-carbon or WMo thermal units [6-8] by means of horizontally directed crystallization. For crystal growing a charge with low content of main impurities was used (see Table 1). During the crystal growing, the impurities in the charge were either evaporated, or pushed off by the inter-phase interface. As a result, the composition of impurities changes gradually from the beginning of the crystal to its ending. Then the disk-like samples of diameter $10 \mathrm{~mm}$ and thickness $1 \mathrm{~mm}$ were cut from the single crystals with subsequent grinding and polishing.

Table 1. Content of impurities in the charge.

\begin{tabular}{lllllllll} 
Element & $\mathrm{Ca}$ & $\mathrm{Cr}$ & $\mathrm{Fe}$ & $\mathrm{Ga}$ & $\mathrm{Mg}$ & $\mathrm{Na}$ & $\mathrm{Si}$ & $\mathrm{Ti}$ \\
\hline $\begin{array}{l}\text { Concentra- } \\
\text { tion, ppm }\end{array}$ & $<5$ & $<2$ & $<4$ & $<3$ & $<2$ & $<10$ & $<15$ & $<10$ \\
\hline
\end{tabular}

The transmission spectra were measured at $T=295 \mathrm{~K}$ by the spectrophotometer of "Specord-UV-VIS" type in the spectral region $200 \div 800 \mathrm{~nm}$. An absorption coefficient $\mathrm{K}(\mathrm{l})$ had been determined after correction by the reflection and unity-thickness normalization were carried out. To measure the TSL curves, the samples were exposed to X-ray irradiation in the air atmosphere at $T=$ $=295 \mathrm{~K}$ during a time period of $70 \mathrm{~min}(\mathrm{Cu}$ anode, $40 \mathrm{kV}, 10 \mathrm{~mA}$ ), then heated with a constant rate of $1 \mathrm{~K} / \mathrm{s}$ up to $T=600 \mathrm{~K}$. TSL signal was received by a photomultiplier FEU-106 operating in the photons-count mode and processed by an automated setup. To distinguish components of the TSL signal, the normalized absorption filters with known transparency band were placed between the sample and the photomultiplier.

\section{Results and discussion}

When describing the obtained results we will proceed from the following well-known regularities of defects' formation in the course of growing the a-sapphire single crystals by means of horizontally directed crystallization in a protective gas ambient of varied reduction potential [8]. A characteristic feature of such a growth is the presence of $\mathrm{O}^{2-}$ anion vacancies (F-centers), which are located in the initial part of the ingot. The concentration of such centers is directly proportional to the reduction potential of gas medium. As to the terminal part of the ingot, the impurities of the extrinsic origin emerge here under the action of crystallization front. The Ti dopant makes the most significant contribution to the optical properties of $\mathrm{a}-\mathrm{Al}_{2} \mathrm{O}_{3}$, while the anion vacancies play a main role in determining the charge state of the Ti dopant $\left(\mathrm{Ti}^{4+}\right.$ or $\left.\mathrm{Ti}^{3+}\right)$. Therefore, the gradient of the vacancies' concentration and that of the Ti dopant, which are directed oppositely, form a complicated non-uniform distribution of defect/dopant complexes determining optical and functional characteristics of the sapphire. This is

$S Q O, 5(4), 2002$ the basis on which we will consider the obtained experimental results.

a) The absorption spectra of sapphire crystals grown in the medium with various reduction potential are shown in Fig. 1. In the spectra of crystals grown in the graphitized-carbon thermal unit in the $\mathrm{CO}$ ambient with low reduction potential, the wide absorption band in the wavelength range $200 \div 230 \mathrm{~nm}$, which is associated with the presence of Ti impurity in $\mathrm{its}^{\mathrm{Ti}^{4+}}$ charge state $[9,10]$, was prevailing (Fig. 1, curves 1 and 2). Similar band was also observed in spectra of crystals grown under neutral conditions, for instance, in the W-Mo thermal unit and vacuum of $P=10^{-4}$ torr (curve 4), or crystals grown by Verneyl's method. In the crystals grown under condition of high reduction potential, the compensation of excessive positive electric charge takes place in accordance with the following reaction: $\left(\mathrm{V}_{\mathrm{o}}^{++}+2 \mathrm{e}\right)+\mathrm{Ti}^{4+} \rightarrow\left(\mathrm{V}_{\mathrm{o}}^{++}+\right.$ $+\mathrm{e})+\mathrm{Ti}^{3+}$, with $\mathrm{V}_{\mathrm{o}}{ }^{++}$being an oxygen vacancy, and $\mathrm{e}-$ an electron. In the absorption spectra of such crystals a band with $\lambda_{\max } \approx 205 \mathrm{~nm}$, that is connected with the presence of considerable concentration (up to $10^{17} \mathrm{~cm}^{-3}$ ) of Fcenters $[10,11]$, was dominating (Fig. 1, curve 3 ). The intensity of this band increased with the gas pressure rise (Fig. 2). It should be noted that under UV irradiation the $\mathrm{Ti}^{4+}$ impurity is reduced by the reaction: $\mathrm{O}^{-2}+\mathrm{Ti}^{4+}+h v \leftrightarrow$ $\mathrm{O}^{-}+\mathrm{Ti}^{3+}$. So, the $\mathrm{O}^{-}$ions appear as a result of the $\mathrm{Ti}^{4+}$ ions reduction, and form centers of coloring, which lead to formation of the wide absorption band with $\lambda_{\max }$ about $400 \mathrm{~nm}$. The $\mathrm{Ti}^{3+}$ ions absorb in the region $500 \div 550 \mathrm{~nm}$, while the peaks of emission of $\mathrm{F}^{+}$-centers, F-centers and $\mathrm{Ti}^{3+}$ ions are located at $335 \mathrm{~nm}, 413 \mathrm{~nm}$ and $720 \mathrm{~nm}$, respectively $[9,10]$.

b) At the temperature interval $295 \div 600 \mathrm{~K}$, the peaks at $T_{\mathrm{m} 1}=425 \mathrm{~K}, T_{\mathrm{m} 2}=440 \mathrm{~K}, T_{\mathrm{m} 3}=480 \mathrm{~K}$ and $T_{\mathrm{m} 4}=560 \mathrm{~K}$

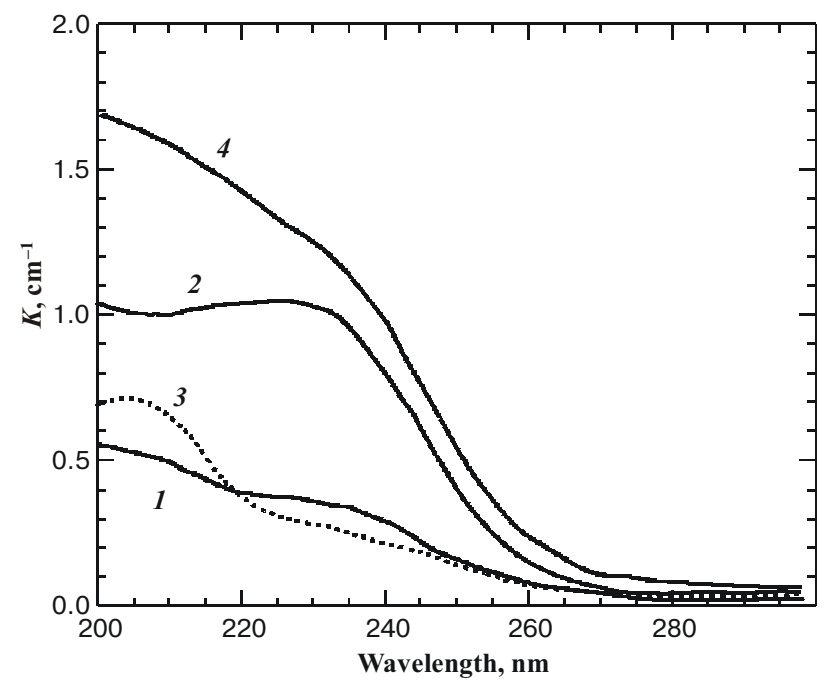

Fig. 1. Absorption spectra of crystals grown in media with low ( 1 , $2,4)$ and high (3) reduction potentials. CO medium: $P=0.05$ torr, beginning ( 1 ) and ending parts (2) of the crystal; Ar+CO medium: $P=10$ torr, terminal section of the crystal (3); $\mathrm{Ar}+\mathrm{CO}$ medium: $P=10^{-4}$ torr, W-Mo thermal unit (4). 


\section{I.V. Blonskyy et al.: Growth conditions influence on thermally ...}

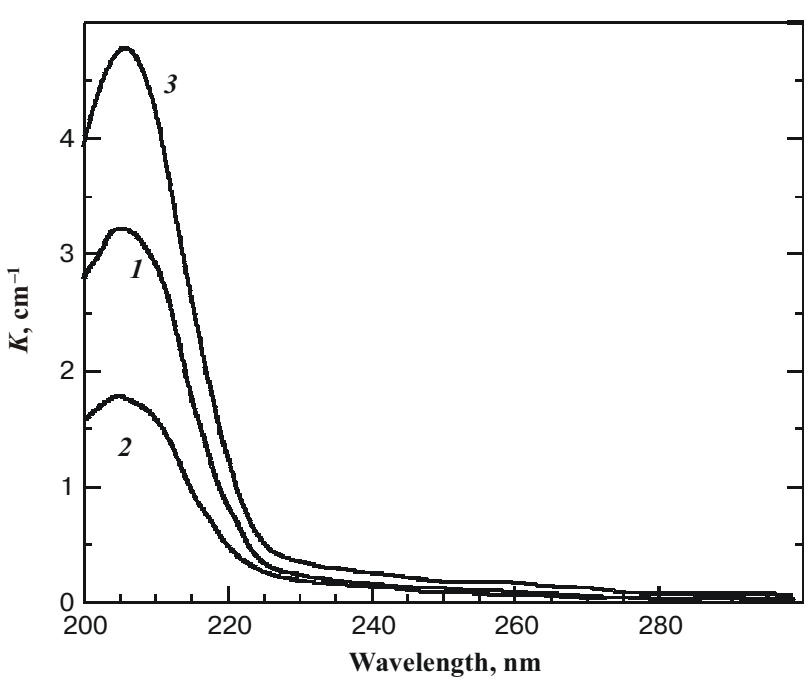

Fig. 2. Absorption spectra of crystals grown in the Ar+CO medium with high reduction potential: $P=10$ torr, beginning part of the crystal ( 1 ); $P=30$ torr, terminal (2) and initial (3) sections of the crystal (3).

were found on the TSL curves of sapphire crystals. The number of peaks and their amplitudes depend on the growth conditions and the position of crystal's section which a sample was cut out from. Two emission bands, one being within the interval $335 \div 480 \mathrm{~nm}$ and another one lying in the wavelength region with $\lambda>560 \mathrm{~nm}$, contribute to the $T_{\mathrm{m} 1}$ peak. In an explicit form this peak is manifested only for crystals grown in media with high reduction potential (Fig. 3, curves 1, 2 and 3). Intensity of this peak increases with the rise of $(\mathrm{Ar}+\mathrm{CO})$ ambient pressure (Fig. 3), as for the F-centers' absorption band. The concentrations of the F-centers in samples 1, 2 and 3, as estimated from the absorption spectra, are $6.0 \cdot 10^{16} \mathrm{~cm}^{-3}$,
$3.4 \cdot 10^{16} \mathrm{~cm}^{-3}$ and $9.2 \cdot 10^{16} \mathrm{~cm}^{-3}$, respectively. Therefore, one can suppose the $T_{\mathrm{m} 1}$ peak of the TSL to be connected with F-centers. For crystals grown in the media of low reduction potential, this peak does not manifest explicitly, being masked by the more intense TSL peak with maximum at $T_{\mathrm{m} 2}=440 \mathrm{~K}$.

The $T_{\mathrm{m} 2}$ peak is predominantly formed by the emission in the region of $\lambda \geq 560 \mathrm{~nm}$ and, for crystals grown in the $\mathrm{CO}$ medium with low reduction potential, this TSL peak was by an order of magnitude more intensive than the other ones (Fig. 4, curve 2). Provided that the reduction potential of the medium rises, the peak intensity is coming down in a manner analogous to that of the $\mathrm{Ti}$ dopant's absorption band, which corresponds to its charge state $\mathrm{Ti}^{4+}$. In particular, for a crystal grown in CO medium under the pressure $P=0.05$ torr, the intensity of the $T_{\mathrm{m} 2}$ peak for a sample cut out from a beginning part of the crystal is as twice as compared to that for the ending-part sample (Fig. 4, curves 1 and 2), that is parallel to the concentration change of $\mathrm{Ti}^{4+}$ ions (Fig. 1, curves 1 and 2). This TSL peak was also observed for the crystal grown in the W-Mo thermal unit in vacuum, i.e. under conditions close to neutral (Fig. 4, curve 3 ). That is why one can suppose this peak to be associated with presence of the $\mathrm{Ti}^{4+}$ ions.

At the temperature region of peak $T_{\mathrm{m} 3}=480 \mathrm{~K}$ the emission with li560 nm was mainly observed. Alike the TSL peak at $T_{\mathrm{m} 1}=425 \mathrm{~K}$, this one is also observed only in crystals grown in media of high reduction potential, and its intensity increases along with the reducing power of a medium (Fig. 3, curves 1, 2, and 3), when Ti dopant is mainly in its electric-charge state $\mathrm{Ti}^{3+}$ [9]. Hence, this TSL peak is likely originated by presence of the $\mathrm{Ti}^{3+}$ ions. Analogously to the $T_{\mathrm{m} 1}$ peak, this one does not also manifest itself explicitly for crystals grown in low-reducing-potential media, that is apparently conditioned by

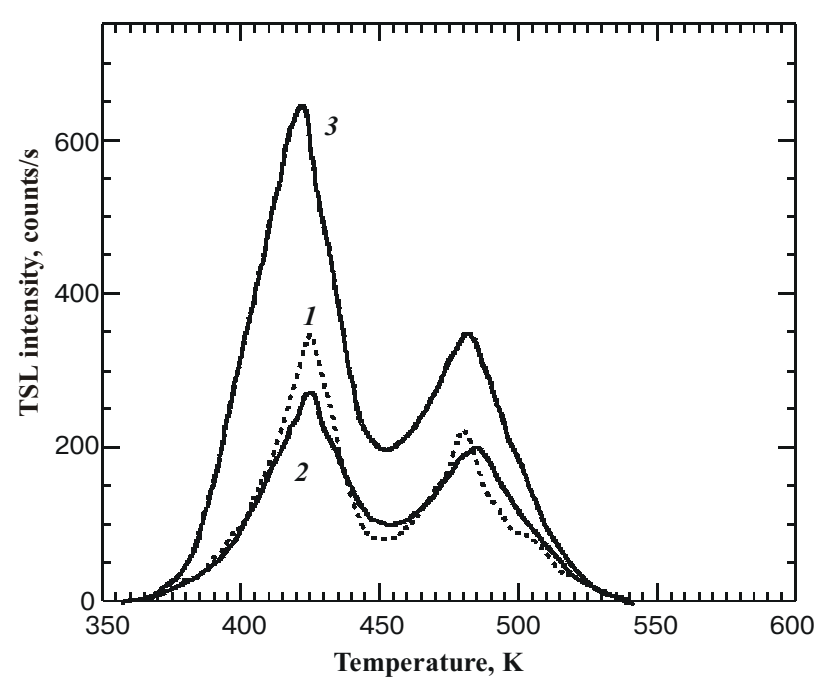

Fig. 3. TSL curves of crystals grown in the $\mathrm{Ar}+\mathrm{CO}$ medium with high reduction potential: $P=10$ torr, initial part of the crystal (I); terminal part of the crystal, $P=30$ torr (2), $P=400$ torr (3).

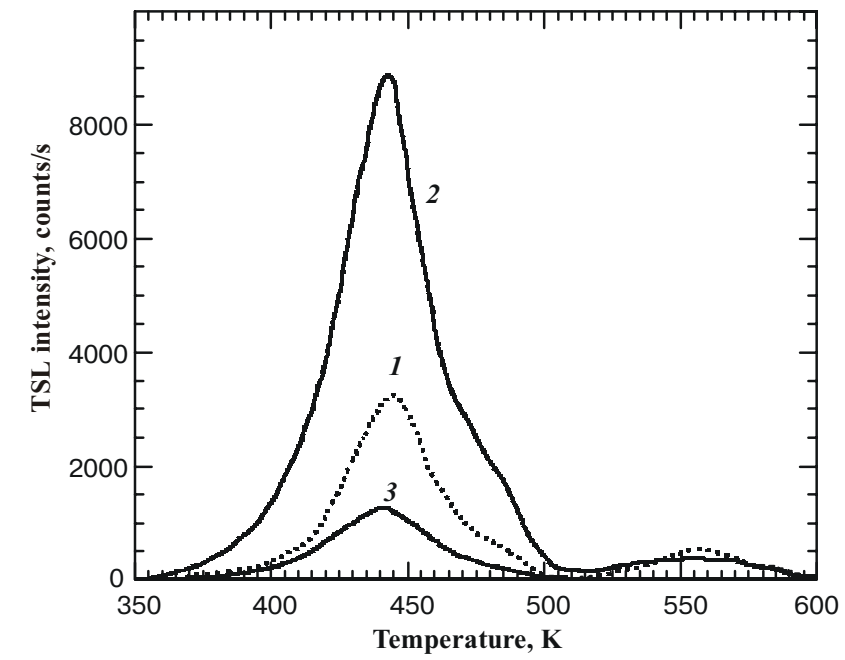

Fig. 4. TSL curves of crystals grown in the $\mathrm{CO}$ medium with high reduction potential: $P=0.05$ torr, beginning ( 1 ) and ending (2) sections of the crystal; vacuum, W-Mo thermal unit (3).

SQO, 5(4), 2002 


\section{I.V. Blonskyy et al.: Growth conditions influence on thermally ...}

the presence of much more intense adjacent TSL peak observed at $T_{\mathrm{m} 2}=440 \mathrm{~K}$.

The TSL peak at $T_{\mathrm{m} 4}=560 \mathrm{~K}$ is formed by the emission region of $335 \mathrm{e} 720 \mathrm{~nm}$. It is observed, along with the $T_{\mathrm{m} 1}$-related peak, for crystals grown in a low-reductionpotential medium, whose absorption spectra are characterized by the $\mathrm{Ti}^{4+}$ ions' band in the range of $200 \div 230$ $\mathrm{nm}$. The behavior of the $T_{\mathrm{m} 4}$-related peak is anticorrelative with that of the $T_{\mathrm{m} 2}$-related peak, namely, the maximal intensity of the $T_{\mathrm{m} 4}$-related peak was achieved for the sample with the minimal intensity of the $T_{\mathrm{m} 2}$-related one (Fig. 4, curves 1 and 2). The $T_{\mathrm{m} 4}$-related peak was not revealed in TSL curves of crystals grown in both the neutral (Fig. 4, curve 3) and high-reduction-potential (Fig. 3) media, i.e. it is connected with simultaneous presence of both the doping and the intrinsic defects of the lattice. Thus, this peak is probably originated from traps of less trivial nature, more likely with the complexes of the type: cathion impurity-anion vacancy.

Therefore, the crystals grown in reducing media of $\mathrm{CO}$ or $\mathrm{CO}+\mathrm{Ar}$ types can be grouped in two following classes with respect to the TSL signal behavior: for crystals grown in high-reduction-potential media, only the $T_{\mathrm{m} 1}$ - and $T_{\mathrm{m} 3}$-related TSL peaks were observed, while for those ones grown in low-reduction-potential media, the $T_{\mathrm{m} 2}$ - and $T_{\mathrm{m} 4}$-related peaks dominate in TSL curves (Figs 3 and 4). This classification is also proved by the results obtained for a crystal grown in the medium with relatively high reduction potential, namely, $\mathrm{Ar}+\mathrm{CO}$ upon $P=10$ torr (see Fig. 5).

The following information is important for data analysis. A composition of the medium has not been monitored during the growing, yet from the results of previous investigations [7] it is known, that under such conditions the concentration of the reducing component $(\mathrm{CO})$ does not decrease, but even increases close to termination of the crystallization. It is also known, that in the terminal

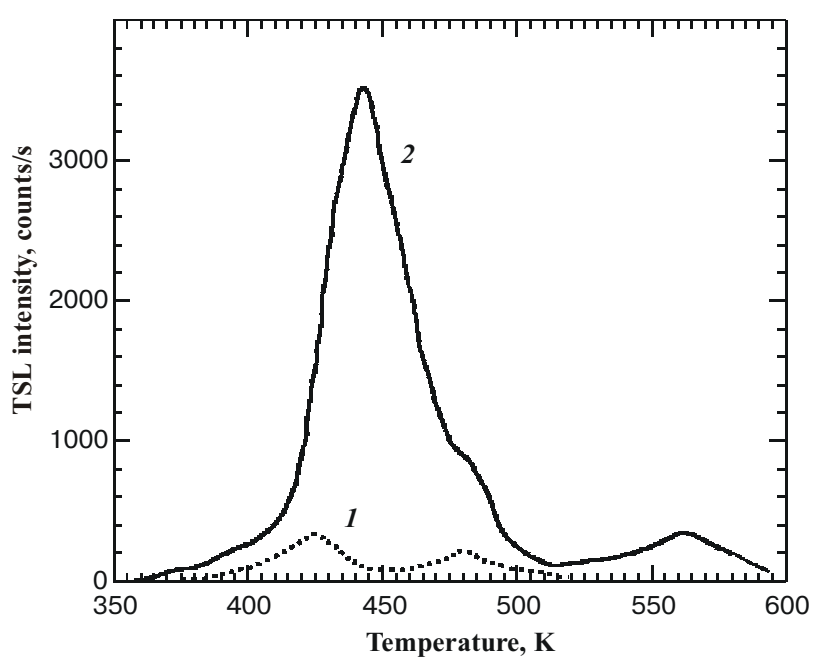

Fig. 5. TSL curves of samples cut from the beginning ( $(1)$ and ending parts (2) of the crystal grown in the $\mathrm{Ar}+\mathrm{CO}$ medium, $P=10$ torr.

$S Q O, 5(4), 2002$ section of a crystal the content of Ti dopant is by 2 or 3 times higher than that in the initial one. It was found that in the absorption spectrum of a sample cut out from the initial part of the crystal, the band associated with Fcenters $\left(\lambda_{\max }=205 \mathrm{~nm}\right)$, was prevailing (Fig. 2, curve 1). For the sample from crystal's central part the intensity of this band was lower, and, accordingly, for the terminal section the characteristic wide band associated with presence of $\mathrm{Ti}^{4+}$ ions, was observed in the region $200 \ddot{2} 230 \mathrm{~nm}$ (Fig. 1, curve 3). Simultaneously, the $T_{\mathrm{m} 1}$ - and $T_{\mathrm{m} 3}$-related TSL peaks were observed for the initial section of the crystal, whereas for the terminal part they were hidden by much more intensive $T_{\mathrm{m} 2}$-related peak (Fig. 5). Besides, the $T_{\mathrm{m} 4}$-related TSL peak emerged for the last case, while it was absent for a sample from the initial section of the crystal.

The phenomena observed may be explained as follows. In the sapphire crystals under consideration, the concentrations of the anion vacancies and $\mathrm{Ti}$ dopants are of the same order of magnitude $\left(10^{17} \mathrm{~cm}^{-3}\right)$ [7]. Seemingly, in the initial part of the crystal owing to the presence of anion vacancies and low Ti concentration, the conditions are provided for effective Ti reduction by the reaction: $\left(\mathrm{V}_{\mathrm{o}}^{++}+2 \mathrm{e}\right)+\mathrm{Ti}^{4+} \rightarrow\left(\mathrm{V}_{\mathrm{o}}{ }^{++}+\mathrm{e}\right)+\mathrm{Ti}^{3+}$. As the Ti concentration increases while one moves towards the terminal section of the crystal, the reduction capability become poorer and the considerable part of Ti dopant remains in the $\mathrm{Ti}^{4+}$ state. It should be noted that lowering of the absorption peak of neutral F-centers is caused with a partial conversion of neutral F-centers into $\mathrm{F}^{+}$centers as a result of the above reaction. It is quite possible that this is the thermally activated charge transfer between closely situated defects (impurity-vacancy pairs) that gives rise to appearance of the $T_{\mathrm{m} 4}$-related TSL peak, which was observed only for the crystals with intermediate degree of the Ti dopant reduction, and was not observed for crystals grown in a neutral medium as well as in crystals with high degree of Ti reduction.

c) Photoluminescence (PL) spectra of five types of sapphire single crystals, each grown with its own specific procedure, have been studied:

type 1 - the crystal is grown in $\mathrm{CO}$ medium under pressure of $0.1 \div 0.3$ torr (reducing atmosphere), not containing microparticles;

type 2 - the crystal is grown in $\mathrm{CO}$ medium under pressure of $0.1 \div 0.3$ torr (reducing atmosphere), containing about $10^{5} \mathrm{~cm}^{-3}$ microparticles;

type 3 - the crystal is grown in $\mathrm{Ar}+10 \% \mathrm{H}_{2}$ medium under pressure of 800 torr (reducing atmosphere), containing about $3 \cdot 10^{6} \mathrm{~cm}^{-3}$ microparticles;

type 4 - the crystal is grown in vacuum of about $10^{-4}$ torr in the W-Mo thermal unit (neutral medium), not containing microparticles;

type 5 - the crystal is grown from a pure raw material in $\mathrm{CO}$ medium of $0.1 \div 0.3$ torr pressure (reducing atmosphere), not containing microparticles.

Photoluminescence has been excited at room temperature in polished disk-shaped sapphire samples (with diameter $20 \mathrm{~mm}$ and thickness $5 \mathrm{~mm}$ ) by the 337-nm radia- 


\section{I.V. Blonskyy et al.: Growth conditions influence on thermally ...}

tion of pulsed 8-ns $\mathrm{N}_{2}$-laser with $5 \mathrm{~kW}$ peak power. To record the spectra in $440 \div 750 \mathrm{~nm}$ spectral range a diffraction spectrometer equipped with CCD array has been used.

Only the samples 2 and 3 have a measurable luminescence under the excitation conditions mentioned above, while the luminescence intensity of the rest samples was negligibly small. The luminescence spectra of the samples 2 and 3 are given in Fig. 6. They look like wide structureless bands in the $450 \div 700 \mathrm{~nm}$ range with peak at $550 \mathrm{~nm}$. It should be emphasized that the samples 2 and 3 contain microparticles. From the absorption spectra of all five types of the samples it follows, that exactly the samples 2 and 3 have a noticeable absorption at 337 $\mathrm{nm}$, which apparently is due to the microparticles presence. The known luminescence bands of F-centers (413 $\mathrm{nm})$ and $\mathrm{Ti}^{4+}$ centers $(425 \mathrm{~nm})$ are situated outside the measurement range. Luminescence bands of $\mathrm{Cr}^{3+}$ center $(690 \mathrm{~nm})$ and $\mathrm{Ti}^{3+}$ centers $(720 \div 770 \mathrm{~nm})$ are much narrower and we have not really detected them. Moreover, all the indicated centers can be excited efficiently only by more short-wavelength radiation at the range $200 \div 250$ $\mathrm{nm}$. Thus it is advisable to interpret the detected emission band as the luminescence of microprecipitates with the estimated size of $1 \ddot{2} 2 \mathrm{mcm}$. The observed twofold difference in luminescence intensity for these two samples can not be easily explained. Really, the sample 2 has twice as high the luminescence signal as the sample 3 has, though the latter features higher microparticle concentration. Apparently we observe the concentration quenching of luminescence caused by the high microparticle concentration.

\section{Conclusion}

Thus, it was established that the TSL curves of sapphire crystals may be sorted into two types depending on the reducing capability of a gas ambient upon growth. For crystals grown in the $\mathrm{CO}$ or $\mathrm{Ar}+\mathrm{CO}$ medium with high reduction potential ( $P \geq 10$ torr), the peaks at $425 \mathrm{~K}$ and $480 \mathrm{~K}$ prevailed in TSL signal. We suppose the origin of these peaks to be connected with presence of the F-centers and $\mathrm{Ti}$ dopant in its $\mathrm{Ti}^{3+}$ charge state. If the crystals were grown in a medium with low reduction potential ( $P \leq 10$ torr) or close-to-neutral medium, then the TSL peaks at $440 \mathrm{~K}$ and $560 \mathrm{~K}$ were dominating. The former peak is most probably associated with the Ti dopant in the $\mathrm{Ti}^{4+}$ charge state, while the latter one is connected with anion defectTi impurity interaction.

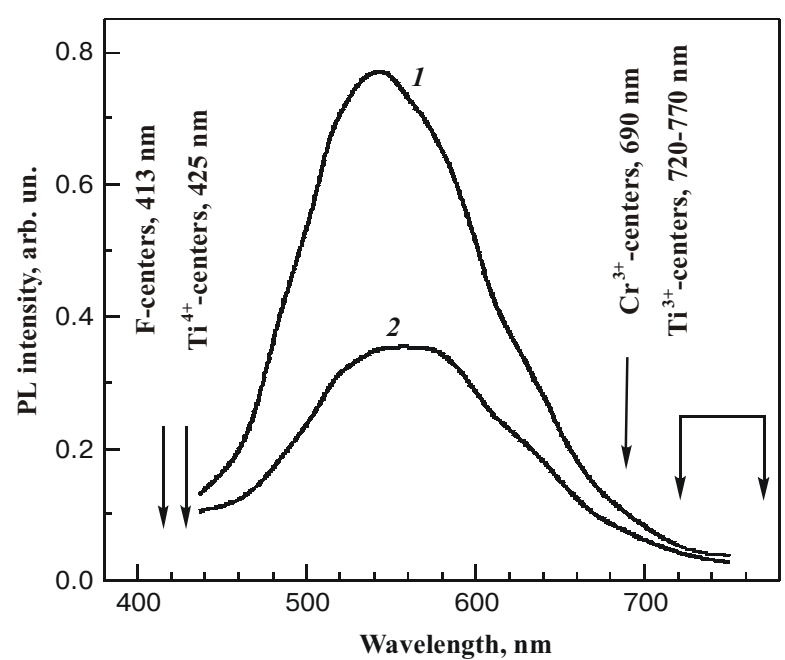

Fig. 6. Photoluminescence (PL) spectra of type 2 sample grown in the $\mathrm{CO}$ medium, $P=0.1-0.3$ torr $(I)$ and type 3 sample grown in the $\mathrm{Ar}+10 \% \mathrm{H}_{2}$ medium, $P=800$ torr (2).

\section{References}

1. F. Ponce and D. Bour // Nature 386, pp. 351-359 (1997).

2. S. Nakamura and G. Fasol, GaN based Light Emitters and Lasers, Springer,Berlin, 256 p.(1997)

3. E.F. Martynovich, V.I. Baryshnikov, V.A Grigorov // Pisma v Zhurn. Teh. Fiz. 11(4), pp. 200-202 (1985) (in Russian).

4. M.S. Akselrod and I.I. Milman // Proceedings of the Academy of Sciences of USSR, Physics Series, 46(12), pp. 23612363 (1982) (in Russian).

5. N.V. Morozov, V.M. Reiterov, P.B. Sergeyev // Quantum Electronics 29(2), pp. 141-144 (1999) (in Russian).

6. A.Ya. Danko, N.P. Katrich, Yu.P. Miroshnikov, V.E. Kachala, G.T. Adonkin // Technique of growing the aluminium oxide single crystals, Patent of Ukraine N 18923A.

7. V.M. Puzikov, A.Ya. Danko, G.T. Adonkin, N.S. Sidelnikova, V.F. Tkachenko, A.T. Budnikov // Semiconductor Physics, Quantum Electronics and Optoelectronics, 3(2), pp. 185-190 (2000).

8. A.Ya. Danko, N.S. Sidelnikova, G.T. Adonkin, A.T. Budnikov, N.P. Katrich, V.N. Kanishchev, S.V. Hizhanovsky, V.M. Puzikov // Functional Materials, NASU Inst. of single crystals, Collection of articles, pp. 200-213 (2001) (in Russian).

9. T.S. Bessonova, M.P. Stanislavsky, V.Ya. Khaimov-Malkov // Problems of atomic Science and Technology, Series FRP and $R M, \mathrm{~N} 1$, pp. 74-80 (1974) (in Russian).

10. G. Molnar, J. Borossay, M. Benabdesselam, P. Iacconi, D Lapraz, K. Suvegh, A. Vertes // Phys. Stat. Sol., A179 (1), pp. 249-260 (2000).

11. B.D. Evans // Phys. Rev., B18 (12), pp. $7089-7098$ (1978). 